\title{
Nanoscale simulations of cement hydrates precipitation mechanisms: Impact on macroscopic self-desiccation and water sorption isotherms
}

\author{
E. Masoero \& I. Shvab \\ School of Engineering, Newcastle University, UK
}

G. Di Luzio

Dipartimento di Ingegneria Civile e Ambientale, Politecnico di Milano, Milan, Italy

G. Cusatis

Department od Civil and Environmental Engineering, Northwestern University, USA

\begin{abstract}
Recent experiments show that the nanoscale morphology of cement hydrates can be tuned via solution chemistry and curing conditions. However, it is not known to what an extent a nano-tailored morphology of cement hydrates may translate into improved macroscale properties. This question is addressed here, focussing on water-content-dependent durability properties, in particular self-desiccation and water sorption isotherms. Nanoparticle-based simulations provide the starting point to create model hydrates structures at the micrometre scale, whose formation mechanisms and resulting morphologies depend on solution chemistry and interaction forces at the nanoscale. These nanoscale mechanisms and morphologies are then used to inform a simple model of cement hydration that predicts pore size distribution, water content, internal relative humidity and thus self-desiccation and water sorption isotherms at the macroscale. The results show that the nanoscale morphology of cement hydrates has indeed an important impact on the above-mentioned durability properties, and that hydrates precipitation in current ordinary cements follows a mechanism that is intermediate between the two frequently used models of homogeneous hydrogelation and boundary nucleation and growth.
\end{abstract}

\section{INTRODUCTION}

The high environmental impact of cement production, alongside its increasing demand, are challenging the target of an $80 \%$ reduction of greenhouse gas emissions by 2050 . This is stimulating empirical research on new chemical formulations, but the industrial deployment of new cements is hindered by the uncertainties that surround their largely untested mechanical performance, especially in relation to long-term durability-related processes such as creep and shrinkage. This is in turn stimulating theoretical research on developing models to predict the engineering performance of a cement paste starting only from knowledge of their chemical composition, curing conditions, and environmental exposure during service. These models are necessarily multiscale, aiming to link the nanometre scale of molecular structures and chemical reactions, with the macroscale of engineering properties (Di Luzio and Cusatis 2009, Davie and Masoero 2015, Bažant et al. 2015, Cusatis et al. 2018).
In the multiscale modelling of cement chemomechanics, a crucial range of length-scales is the "mesoscale" between $1 \mathrm{~nm}$ and $1 \mu \mathrm{m}$. These are the length-scales at which the cement hydrates, precipitating from an aqueous solution during hydration, start to organise themselves into a nanoporous structure. The resulting network of nanopores controls largely the macroscopic interaction between cement paste and water, which is critical for the long-term mechanics (Jennings et al. 2013). Experimental research has shown that, depending on solution chemistry and curing conditions, cement hydrates with similar chemical compositions can precipitate into a wide range of sub-micrometre structures with different morphologies and nanopore networks. This hasbeen shown in particular for calcium-silicate-hydrate $(\mathrm{C}-\mathrm{S}-\mathrm{H})$, which is the main binding phase in portland cement pastes (Tajuelo Rodriguez et al. 2015). The implication is that it may be possible to tune the sub-micrometre structure of cement paste and concrete in order to maximise the engineering 
properties at the macroscale. However, this vision can be realised only by: (i) developing new theoretical tools to guide the experiments- these tools should help identify chemical formulations and curing conditions that may lead to the most desirable nanoscale features; (ii) proving that the submicrometre, nanoporous structure of $\mathrm{C}-\mathrm{S}-\mathrm{H}$ and other hydrates actually has a significant impact on the macroscopic properties of a paste.

This paper is divided in two main sections addressing the two challenges mentioned just above. First, results from recent nanoparticle simulations of $\mathrm{C}-\mathrm{S}-\mathrm{H}$ precipitation are presented (Shvab et al. 2017), focussing on two precipitation mechanisms: (i) radial growth of compact, emispherical, mesoporous domains of $\mathrm{C}-\mathrm{S}-\mathrm{H}$, which is the nanoscale version of the Boundary Nucleation and Growth (BNG) mechanism (Thomas 2007), and (ii) precipitation and aggregation of $\mathrm{C}-\mathrm{S}-\mathrm{H}$ nanoparticles (hydrogelation) (Ioannidou et al. 2014). The mesoscale morphology evolutions resulting from these two different mechanisms are then taken as inputs for a simple model of cement hydration at the macroscale, which predicts the evolution of porosity and self-desiccation in the cement paste (Masoero et al. URev). The results of the combined simulations indicate that the experimentally observed self-desiccation of low-alkali cement pastes can be captured by a nanoscale precipitation mechanism that is intermediate between BNG and hydrogelation. The impact of the different precipitation mechanisms and nanoscale morphologies on the water sorption isotherm is also presented and discussed.

\section{METHODS}

\subsection{Nanoparticle simulations of precipitation}

In the last decade, simulations of nanoparticle nucleation and aggregation have started to clarify the relationship between mesoscale morphology and experimentally observed structural features and mechanical properties at the sub-micrometre level (González-Teresa et al. 2010, Masoero et al. 2012, Masoero et al. 2013, Masoero et al. 2014, Ioannidou et al. 2016). Only very recently, though, first nanoparticle simulations have been developed where the rates of particle nucleation and dissolution are quantitatively related to the chemical composition of the surrounding aqueous solution and to the mechanical interaction energy between particles (Shvab et al. 2017). These simulations provide a new framework to incorporate key experimental variables, such as solution chemistry and curing conditions, into the nanoparticle simulations. Only several key features of such simulations will be presented here, in order to let the reader appreciate the origin of the nanoscale morphologies presented
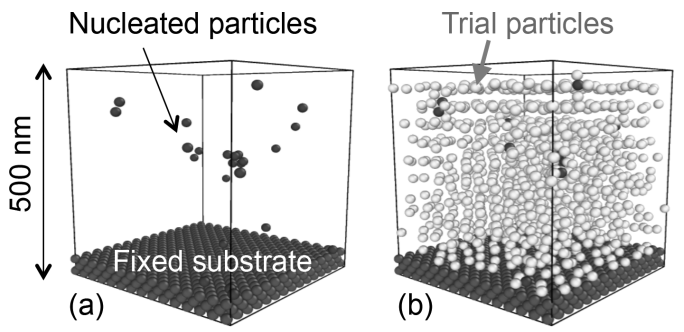

Figure 1. (a) Nanoparticle simulation box containing a fixed substrate and several nucleated particles. (b) Same configuration with also $\mathbf{M}$ trial particles for nucleation visualized.

later. The interested reader can find more details in (Shvab et al. 2017).

The simulations in (Shvab et al. 2017) are based on the Kinetic Monte Carlo (KMC) method. The starting configuration is an empty simulation box, of characteristic size of $c a .500 \mathrm{~nm}$ (see Figure 1). The box is periodic in the horizontal plane, closed at the bottom by a disordered layer of nanoparticles representing a surface of tricalcium silicate $\left(\mathrm{C}_{3} \mathrm{~S}\right.$ in cement notation, with $\mathrm{C}=\mathrm{CaO}$ and $\mathrm{S}=\mathrm{SiO}_{2}$ ), and with a closed fixed boundary at the top. The particles forming the bottom layer are kept fixed during the simulation, i.e. they cannot dissolve nor move, but they do interact mechanically with the $\mathrm{C}-\mathrm{S}-\mathrm{H}$ particles that nucleate and aggregate inside the box.

The KMC simulations consist of a series of events, which can be the nucleation of a new $\mathrm{C}-\mathrm{S}-\mathrm{H}$ particle or the deletion of an existing one. The $\mathrm{C}-\mathrm{S}-\mathrm{H}$ particles have fixed diameter of $c a .10 \mathrm{~nm}$, with a small polydispersity to avoid crystallisation. The chemistry of the solution is fixed and, for the purpose of this paper, it is entirely described by its supersaturation $\beta$ with respect to $\mathrm{C}-\mathrm{S}-\mathrm{H}$ precipitation. The simulations presented later will consider two scenarios: one with $\beta$ fixed in time, and the other with $\beta$ decreasing with time following a realistic evolution for cement hydration from the literature (Bullard et al. 2015). In reality, the evolution of $\beta$ is determined by the relative rates of $\mathrm{C}-\mathrm{S}-\mathrm{H}$ precipitation and $\mathrm{C}_{3} \mathrm{~S}$ dissolution, but here for simplicity $\beta(t)$ is given directly as an input and $\mathrm{C}_{3} \mathrm{~S}$ dissolution is not simulated: only $\mathrm{C}-\mathrm{S}-\mathrm{H}$ precipitation is considered. The simulations also do not account for: partial growth or dissolution of the $\mathrm{C}-\mathrm{S}-\mathrm{H}$ particles, anisotropy of the $\mathrm{C}-\mathrm{S}-\mathrm{H}$ particles, phases other than $\mathrm{C}-\mathrm{S}-\mathrm{H}$ (for example, calcium hydroxide), or diffusion of ions in solution leading to a spatial field of supersaturation. All these features could be incorporated in the simulations, but for now $\mathrm{C}-\mathrm{S}-\mathrm{H}$ nucleation/deletion will suffice to obtain several mesoscale morphologies to inform a larger-scale hydration model. 
At each step, the KMC algorithm selects the next event that takes place from a list of all possible $\mathrm{C}-\mathrm{S}-\mathrm{H}$ nucleation and deletion events. Building this list can be tricky. If one considers a generic state with $N$ C-S-H particles already nucleated, then the complete list will certainly include $N$ possible deletion events (each $\mathrm{C}-\mathrm{S}-\mathrm{H}$ particle can be deleted). For the list of possible nucleation events, instead, one should consider the nucleation of a new $\mathrm{C}-\mathrm{S}-\mathrm{H}$ particle in every possible position, and there is an infinity of those. To overcome this problem, one can (Shvab et al. 2017):

1. discretise the simulation box with a rectangular lattice of $\mathrm{M}$ sites;

2. place only one trial particle in each lattice site;

3. search for a minimum of interaction energy $U$ between each trial particle and the other $\mathrm{N}$ existing particles, by letting the generic trial particle sample different positions only within its lattice cell.

This approach ensures convergence of the overall precipitation mechanisms and precipitation rates, as the lattice spacing is reduced, viz. as $\mathbf{M}$ is increased (Shvab et al. 2017).

Once the list of $N$ possible deletion event and $M$ possible nucleation events is constructed, the KMC requires that a rate is associated to each of those events. (Shvab et al. 2017) proposed coarse-grained expressions for the rates of particle nucleation and deletion that are based on the assumption of a mechanism of classical growth at the molecular scale. According to this molecular mechanisms, a particle nucleation event implicitly represents a single molecule of $\mathrm{C}-\mathrm{S}-\mathrm{H}$ to which a series of $n$ other molecule attach in all radial directions, until the desired diameter of $c a$. $10 \mathrm{~nm}$, is reached (the opposite for particle deletion). By modelling each of the $n$ molecular reactions with Transition State Theory (Lasaga 2014), the resulting effective rates become:

$$
\begin{aligned}
& R^{i n}=k r_{0}^{*} \beta a^{2}\left\{\sum_{i=1}^{n} \exp \left(\chi \frac{\gamma_{i} \Delta \Omega_{i-1}^{i}+\Delta U_{i-1}^{i}}{k_{B} T}\right)\right\}^{-1}, \\
& R^{d e l}=r_{0}^{*} a^{2}\left\{\sum_{i=n}^{1} \exp \left[(1-\chi) \frac{\gamma_{i} \Delta \Omega_{i}^{i-1}+\Delta U_{i}^{i}}{k_{B} T}\right]\right\}^{-1},
\end{aligned}
$$

$R^{i n}$ and $R^{d e l}$ are respectively the nucleation and deletion rate of a generic particle. They both depend on:

- $r_{0}^{*}$, which is the intrinsic rate of the basic chemical reaction driving precipitation and dissolution. For the case of $\mathrm{C}-\mathrm{S}-\mathrm{H}$ with calciumto-silicon ratio of 1 , this reaction may be:

$$
\mathrm{Ca}^{2+}+\mathrm{H}_{2} \mathrm{SiO}_{4}^{2-}+3 \mathrm{H}_{2} \mathrm{O} \rightleftharpoons \mathrm{C}-\mathrm{S}-\mathrm{H}
$$

- $a$, which is characteristic the size of a $\mathrm{C}-\mathrm{S}-\mathrm{H}$ molecule (ca. $0.65 \mathrm{~nm}$ );

- $n$, which is the number of $\mathrm{C}-\mathrm{S}-\mathrm{H}$ molecules to be added in a generic radial direction to go from one $\mathrm{C}-\mathrm{S}-\mathrm{H}$ molecules to the radius of a $\mathrm{C}-\mathrm{S}-\mathrm{H}$ nanoparticle (here, $c a .5 \mathrm{~nm}$ );

- $\gamma$, which is the interfacial energy between solid $\mathrm{C}-\mathrm{S}-\mathrm{H}$ and aqueous solution;

- $k_{B} T$, which is the product between Boltzmann constant and temperature in Kelvin degrees;

- $\Delta \Omega$, which is the change of particle surface when its radius goes from size $\mathrm{n} \cdot(\mathrm{i}-1)$ molecules to size $\mathrm{n} \cdot \mathrm{i}$ molecules in $R^{\text {in }}$, vice versa in $R^{d e l}$,

- $\Delta U$, which is the change in total interaction energy in the system caused by the radius of the considered particles increasing or decreasing by one molecule. In (Shvab et al. 2017), this change in energy is simplified and expressed as a direct function of the change in energy caused by inserting or deleting a full particle. Of course, $\Delta U$ depends on the spatial locations of a particle, and the process of local minimisation described at point 3 above impact this value in the rates.

Besides these common features, $R^{\text {in }}$ also depends on the supersaturation $\beta$ of the solution and on a prefactor $k$ which is directly proportional to the volume of the lattice cell associated to the trial particle being considered for insertion. Finally the $\chi$ factor, which can be between 0 and 1 , quantifies how much one should consider the intermediate transition state, between molecules in solution and solid molecule, to be already solid in terms of displaying surface energy and mechanical interactions. Classical Nucleation and Growth Theory (CNGT) assumes $\chi=1$, hence $\Delta \Omega$ and $\Delta U$ matter only for the dissolution rate. However, CNGT is not meant to describe series of chemical reactions, nor it consider explicitly insertion and deletion, but rather the net rates of nucleation given by $R^{\text {in }}$ minus $R^{d e l}$. If one assume $\chi=1$ in the KMC simulations proposed here, then particles could form on top of existing ones (because their very unfavourable interaction $\Delta U$ would be neglected in $R^{i n}$ ), but at the very next step the same particle would be immediately deleted due to the very large $\Delta U$ in $R^{d e l}$. Overall this would result in very inefficient calculations and unphysical kinetics. Therefore, we prefer to use instead an intermediate value of $\chi=1$, which is analogous to what is usually done in the field of non-Newtonian fluid mechanics (Shvab et al. 2017).

Once the list of rates is known for all N+M possible events, the KMC algorithms selects one event randomly, with a probability that is proportional to its rate. The event is realised, thus a particle is inserted at a specific location or deleted, and the 
time is advanced in proportion to the inverse of the cumulative rate of all $\mathrm{N}+\mathrm{M}$ possible events.

\subsection{Macroscale hydration model}

The nanoscale simulations described in the previous section provide possible morphological evolutions of $\mathrm{C}-\mathrm{S}-\mathrm{H}$ domains, precipitating from solution during cement hydration. The model in this section will take those morphologies as inputs and predict their impact on the macroscopic processes of self-desiccation during cement hydration and water sorption isotherms.

Self-desiccation is the drop of internal relative humidity (iRH) in a cement paste caused by the consumption of water, due to its reaction with $\mathrm{Ca}$ and $\mathrm{Si}$ to produce $\mathrm{C}-\mathrm{S}-\mathrm{H}$ (eq 3 ) and calcium hydroxide. Water sorption isotherms instead quantify the amount of absorbed water when the sample is equilibrated with an environment at given relative humidity $(\mathrm{RH})$. Both self-desiccation and sorption isotherms, therefore, involve the relationship between water content and RH. For pores larger than 1-2 nanometres, such relationship can be quantified using the Kelvin equations, which relates the size of the largest saturated pore with the $\mathrm{RH}$.

The model in this section relates the degree of hydration (DOH) of a cement paste with: (i) the amount of consumed water, assuming hydration in sealed conditions; (ii) the total volume of pores in the paste, decreasing with the $\mathrm{DOH}$, and (iii) the evolving pore size distribution. Assuming equilibrium conditions, one can thus compute the size of the largest saturated pore and thus the corresponding internal $\mathrm{RH}$, to predict self-desiccation. Furthermore, at each $\mathrm{DOH}$, one can use the pore size distribution and compute the amount of water that would be adsorbed at each RH between 0 and 1 , viz. the water sorption isotherm. This section will present only an overview of the most important features of this model. The interested reader will find more details in a dedicated article by the same authors as this one, currently under review (Masoero et al. URev).

The hydration model starts from a statistical description of $\mathrm{C}_{3} \mathrm{~S}$ grains suspended in water, similar to (Masoero et al. 2014). The solution-filled space between the grains is assumed to be discretised with cylinders of height $D / 2$ and diameter $D$, which represent the capillary pores (see Figure 2). The lateral surface of the cylinders is in contact with $\mathrm{C}_{3} \mathrm{~S}$ grains, thus the total lateral surface of all cylinders must equal the surface area of the cement paste. The initial size distribution of the capillary pores is entirely described by the size distribution of these cylinders, which can be assumed to be a power-law with many small cylinders, and few large

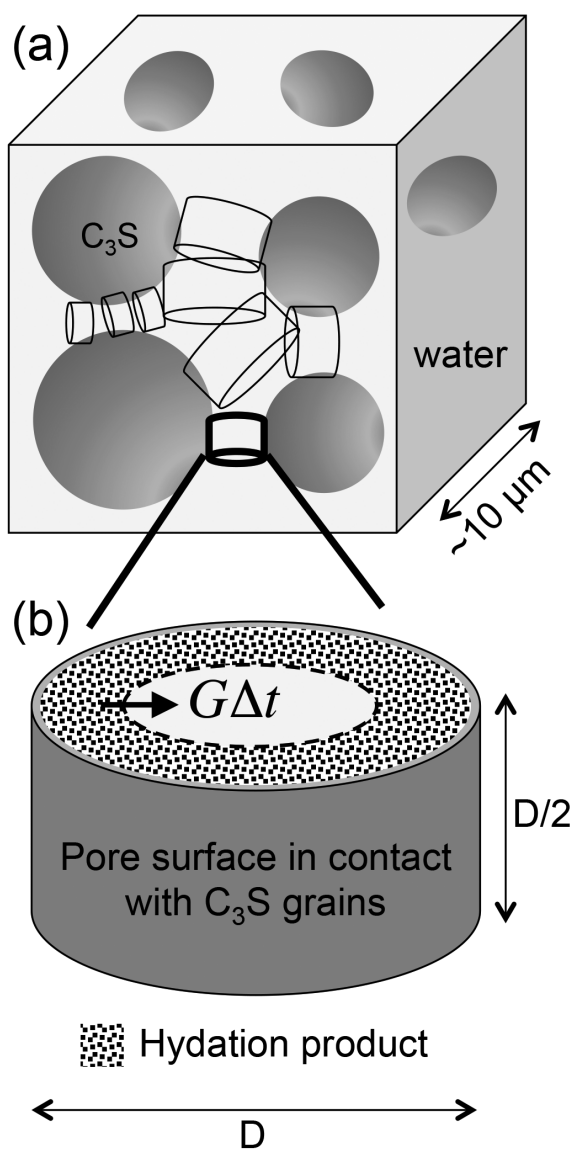

Figure 2. (a) Suspension of cement grains in water, and discretisation of the space between grains using cylinders with different sizes. (b) Formation of hydration product in a pore.

ones, in order to represent flocculation of the $\mathrm{C}_{3} \mathrm{~S}$ grains. Fixing the minimum and maximum $D$ to reasonable values, e.g. $D=1 \mu \mathrm{m}$ as minimum size (a typical resolution of cement hydration models) and $\mathrm{D} \approx 10 \mu \mathrm{m}$ as maximum size, the cylinder size distribution is fully determined by two variables: the total number of cylinders, and the exponent of the power law. These two variables can be determined in order to match the surface area of the cement grains and the water-to-cement ratio, both of which are typical design variables for a cement paste.

The model always assumes stoichiometric balance between reactants (water and $\mathrm{C}_{3} \mathrm{~S}$ ) and hydration product ( $\mathrm{C}-\mathrm{S}-\mathrm{H}$ and calcium hydroxide). The hydration product grows radially starting from the outer surface of each cylindrical pore inward, with a linear growth rate $G$ (see Figure $2 b$ ). Upon 
increasing the hydration time by $\Delta t$, a certain volume of hydration product is produced in each cylinder, depending on its size (small cylinders may get entirely filled with product, stopping precipitation there). This causes a change of capillary pore size distribution over $\Delta t$, since the remaining capillary pores are only the inner parts of the partially filled cylinders. The volume of newly formed hydration product can be converted into mass and, by stoichiometric balance, into mass of dissolved $\mathrm{C}_{3} \mathrm{~S}$ and of consumed water, thus providing the change in DOH as well as the new water content of the paste. However, converting from volume of hydration product into mass requires knowledge of the nanopore volume per unit volume inside the hydration product: this is where the nanoscale morphologies from the previous section are needed as input.

The hydration model allows considering a solid volume fraction ( $\eta$, complement to 1 of the nanopore volume fraction) that is either fixed and constant in time, or increasing in time from a minimum to a maximum value. Since the above-mentioned change of DOH during $\Delta t$ depends both on $G$, on $\eta$ of the newly formed hydration product and, if densification is assumed, on the change of $\eta$ of the previously precipitated product, it is possible overall to quantify these parameters and their evolution in time in order to match the experimentally observed hydration rate, e.g. measured from calorimetry (Bullard et al. 2011). In this manuscript, however, we will only consider the relationship between $\mathrm{DOH}$ and $\mathrm{RH}$ (via the water content and pore size distribution). In such a case, time is just an internal variable for the simulation to progress, but the actual timescale will not influence the results that depend only on "configuration" and not on time.

The last point to clarify is how the average solid fraction $\eta$ is related to the size distribution of the nanopores inside the hydration product. In principle, one could measure the pore size distribution directly from the morphologies in the previous section. For now, however, it is sufficient to interpolate and extrapolate pore size distributions that have already been published for homogeneously nucleated particle aggregates with $\eta=0.33$ and 0.52 (Ioannidou et al. 2016). The details of the interpolation and extrapolation will be given elsewhere (Masoero et al. URev).

Overall, the hydration model outlined here provides a series of $\mathrm{DOH}$ and, for each of them, the total volume of capillary pores, the total volume of nanopores in the $\mathrm{C}-\mathrm{S}-\mathrm{H}$ gel, the total volume of water, and the size distributions of both capillary and gel pores. With these data, one can compute self-desiccation and water sorption isotherms as functions of the $\mathrm{DOH}$, for a paste with given water-to-cement ratio and specific surface area of the grains, and for a given nanoscale morphology of the $\mathrm{C}-\mathrm{S}-\mathrm{H}$.

\section{RESULTS}

\subsection{High-density and low-density $\mathrm{C}-\mathrm{S}-\mathrm{H}$ morphologies from nanoscale simulations}

Figure 3 shows two different mesoscale mechanisms of precipitation obtained with the nanoparticle-based KMC simulations presented in section 2.1. Both simulations use a pairwise Lennard-Jones interaction potential, with interaction strength $\varepsilon$, which quantifies the amount of energy required to completely separate two particles that were originally in contact.

The $\mathrm{BNG}$ mechanism in Figure $3 \mathrm{a}$ emerges when: (i) $\varepsilon$ is in the order of the solid-solution surface energy $\gamma$ multiplied by the cross sectional area of a particle, and (ii) the interaction between nucleated particles andparticles forming the substrate is slightly weaker than the interaction between nucleated particles only. Under such conditions, the first particle nucleates on the substrate, whereas subsequent particle nucleate on top of other previously nucleated particles. This leads to the hemispherical growth of a well-packed domain of nanoparticles, which is typical of a BNG mechanism. The local density inside the precipitated domain is always high (random close packing), and not changing during the precipitation process.

The evolution of precipitation rate corresponding to this BNG mechanism is shown in Figure 3b, for two different evolutions of the solution chemistry. In one case, the supersaturation of the solution with respect to $\mathrm{C}-\mathrm{S}-\mathrm{H}$ precipitation, $\beta$, is assumed to change in time, following a decreasing trend that is typical of bulk cement solutions (Bullard et al. $2015)$. In the other case, $\beta$ is assumed to stay constant in time, which may be a realistic scenario in the vicinity of the interface between unhydrated cement and solution, viz. near the substrate in the simulations (Del Gado et al. 2014). Figure 3b shows that a decreasing $\beta$ leads to an initial acceleration of the precipitation process, followed by deceleration: this is the typical trend of hydration rate expected for ordinary cement pastes (Bullard et al. 2011). By contrast, assuming a constant $\beta$ leads to an unrealistic rate evolution, with initial acceleration followed by a constant rate.

The homogeneous mechanism in Figure 3c emerges when one considers much weaker interactions compared to the BNG case (approximately 100 times weaker). Under such conditions, the influence of the interaction energy $U$ on the rate is small, and particles tend to nucleate everywhere in the solution, to then aggregate a bit. As a result, 

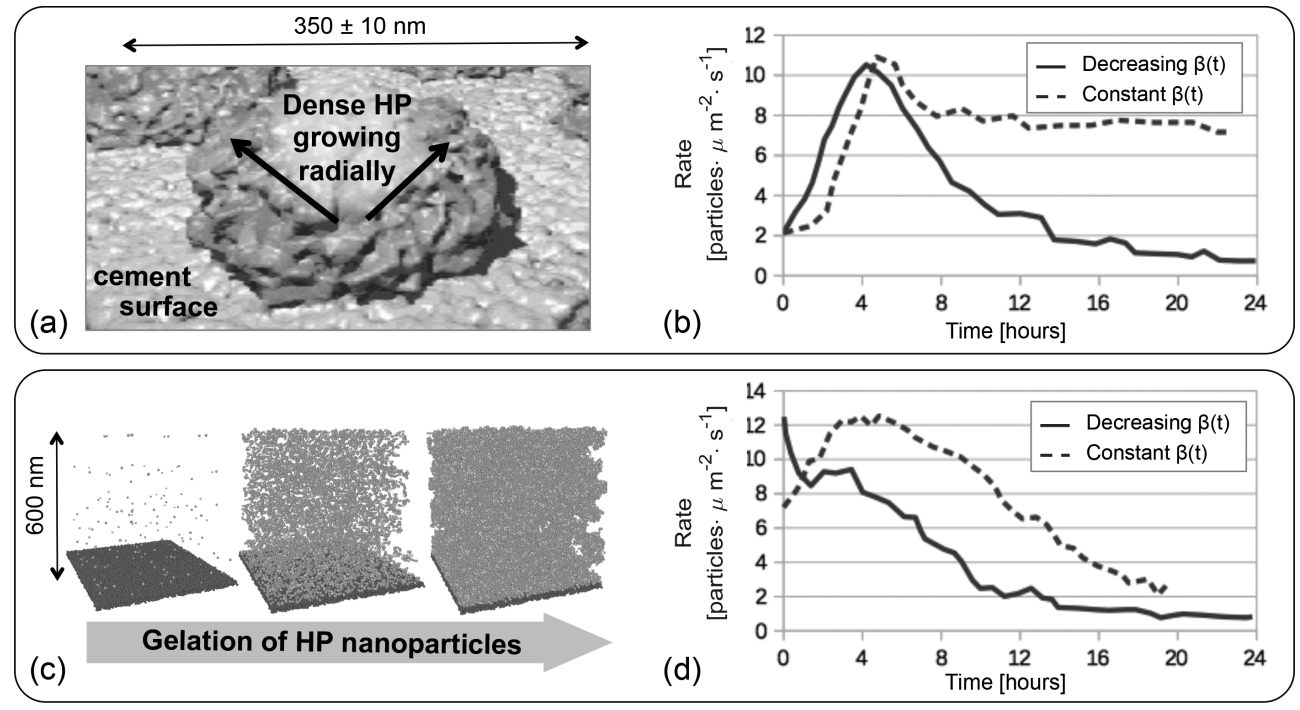

Figure 3. (a) Boundary Nucleation and Growth (BNG) precipitation mechanism, obtained when particle-particle and particle-substrate interactions are strong. (b) Predicted rates corresponding to the BNG mechanism: cases for a solution supersaturation $\beta$ that decreases with time, as typical for bulk cement solutions, or constant in time. (c) Homogeneous nucleation mechanism, obtained when the interaction forces are weak. (d) Predicted rates corresponding to the homogeneous mechanism, again for decreasing and constant $\beta$.

the precipitated domain immediately covers the entire simulation box and the local density, which was always high in the BNG model, is now increasing with time within the precipitated domain. This densification has implications that will be important for the discussion of self-desiccation and sorption isotherms later in this paper.

The precipitation rate corresponding to the homogeneous mechanism is shown in Figure 3d. A $\beta$ decreasing with time leads to an alwaysdecreasing rate, without initial acceleration, in contrast with the experiments that always show acceleration. This happens because the interaction energy $U$ is small and therefore the nucleation rate in eq 1 is governed by the temporal evolution of $\beta$. If $\beta$ is kept constant, the rate shows some sign of initial acceleration and subsequent deceleration: the initial acceleration in this case is due to the weak aggregation processes that take place homogeneously in the solution. In any case, the homogeneous precipitation mechanism entails that the number of precipitated particles per unit of substrate is proportional to the space available for nucleation in the direction perpendicular to the substrate. In other words, the rate in Figure $3 d$ increases linearly with the height of the simulation box, and this entails a proportionality between rate and water-cement ration that is not observed in the experiments (Shvab et al. 2017). The only way to reconcile the homogeneous "gelation" mechanism with the experimental results is to assume that the precipitation of $\mathrm{C}-\mathrm{S}-\mathrm{H}$ is confined to a limited region near the surface of the cement grain (Del Gado et al. 2014), a so-called "reaction zone" that several authors have quantified in the range of $400 \mathrm{~nm}$ to $1 \mu \mathrm{m}$ (Masoero et al. 2014), consistent with the $600 \mathrm{~nm}$ box considered here.

\subsection{Self-desiccation as a function of nanoscale morphology}

Figure 4 shows the self-desiccation predicted by the hydration model in section 2.2. Three mechanisms of nanoscale morphology evolution are considered, and their impact on self-desiccation is compared with an experimental result for a low-alkali cement paste with water-to-cement ratio of 0.4 , Blaine fineness (cement surface area) of $400 \mathrm{~m}^{2} / \mathrm{g}$, and hydrating in sealed conditions (Muller et al. 2013, Muller 2014). The considered nanoscale mechanisms are: (i) the BNG mechanism from Figure $3 \mathrm{a}$, modelled by considering that the hydration product in Figure $2 \mathrm{~b}$ precipitates immediately with solid fraction $\eta=0.655$, constant in time; (ii) the homogeneous (gelation) mechanism in Figure 3c, modelled by considering that hydration product initially forms with very low solid fraction $\eta=0.01$, which increases linearly with time towards a maximum value of 0.74 ; (iii) a case intermediate between the previous two, viz. a BNG 


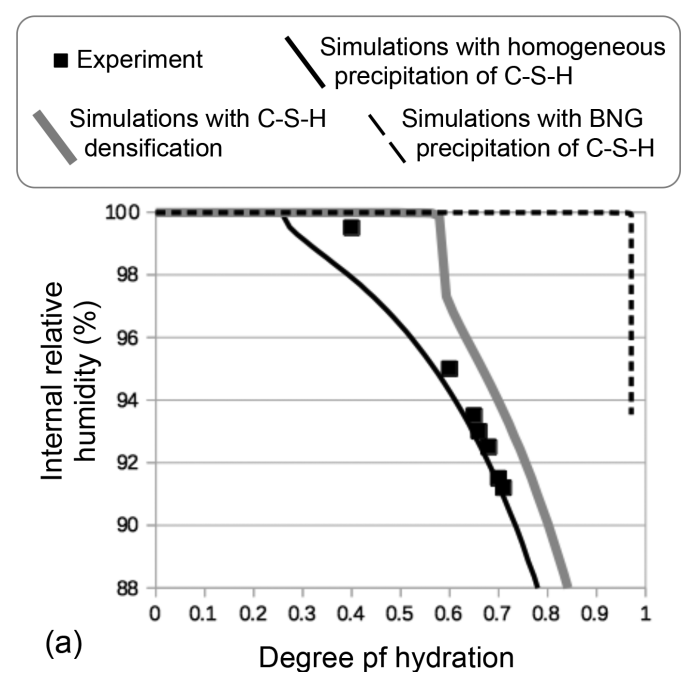

Figure 4. Self-desiccation of a low-aalkali paste hydrating in sealed conditions: comparison between experiment and simulations with different assumptions regarding the precipitation mechanisms of $\mathrm{C}-\mathrm{S}-\mathrm{H}$ at the sub-micrometre scale.

mechanisms where $\eta$ increases in time from a minimum of 0.195 to a maximum of 0.74 . This last mechanisms is indeed suggested by nuclear magnetic resonance measurements of $\mathrm{C}-\mathrm{S}-\mathrm{H}$ density on the same paste as in the self-desiccation experiment (Muller et al. 2013, Muller 2014).

The results in Figure 4 show that the BNG mechanisms largely overestimate the degree of hydration at which the internal relative humidity starts to decrease: $c a$. 0.99 compared to the $c a .0 .4$ from the experiment. The reason is that the Kelvin equation predicts a significant drop of humidity only when pores of $c a$. $100 \mathrm{~nm}$ or smaller get desaturated. A large mass of $\mathrm{C}-\mathrm{S}-\mathrm{H}$ in needed before the dense hydration product of the BNG mechanism fills the capillary pores to the extent of leaving only pores smaller than $100 \mathrm{~nm}$ saturated.

By contrast, the homogeneous gelation mechanism implies that the whole capillary porosity is immediately filled with a very-low-density hydration product. This product has nevertheless an internal pore network with sub-micrometre average pore size (see Figure 3c). As water is consumed during hydration, some of these small pores are immediately desaturated and, according to Kelvin equation, the internal relative humidity starts to drop at degrees of hydration well below the experimental 0.4. The prediction with this gelation mechanism gets increasingly similar to the experimental data while the degree of hydration increases. This is because at 0.4 degree of hydration, the experimental pastes have most of their capillary pores filled, which is the same conditions described by the gelation mechanism. Thus, from degree of hydration 0.4 onward, the configuration of filled capilary pores with a densifying gel in them, represented by the gelation mechanism, is realistic. On the other hand, the gelation mechanism still entails the issue of predicting a too-early onset of self desiccation and a linear dependnece of the degree of hydration on the water-to-cement ratio (see section 2.1).

The intermediate mechanism (low-density BNG plus densification) leads to results that are indeed intermediate between the homogeneous and BNG mechanisms. The degree of hydration corresponding to the onset of self-desiccation is overestimated compared to the experiment: 0.6 instead of 0.4 . However, the issue related to a dependence on the water-to-cement ratio is removed, and overall the prediction of self-desiccation is satisfactory, especially if one considers the uncertainties related to the very simplistic hydration models employed in this work.

\subsection{Water isotherms as functions of nanoscale morphology}

Figure 5 shows water sorption isotherms at two different degrees of hydration (0.1 and 0.75$)$ for simulations with the three mechanisms of nanoscale precipitation already discussed in relation to the self-desiccation results in Figure 4. In all cases, the isotherms show an increase of absorbed water with increasing degree of hydration for $\mathrm{RH} \leq 0.99$, whereas a closer look at Figure 5 shows that the final absorbed water at $\mathrm{RH}=1$ decreases with increasing degree of hydration. The reason for these trends is that the Kelvin equation associates the water adsorbed at $\mathrm{RH} \leq 0.99$ with pore diameter of $c a .100 \mathrm{~nm}$ or less, which are mainly

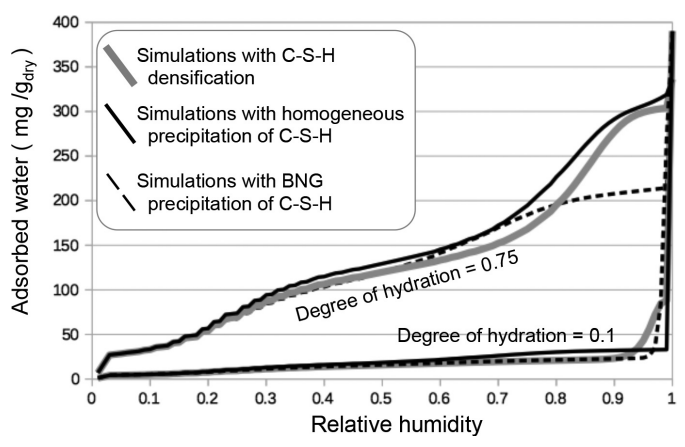

Figure 5. Water sorption isotherms for model cement pastes whose hydration has been simulated using different assumptions regarding the nanoscale mechanisms of $\mathrm{C}-\mathrm{S}-\mathrm{H}$ precipitation. 
inside the $\mathrm{C}-\mathrm{S}-\mathrm{H}$ gel. An increasing degree of hydration corresponds to an increasing volume of C-S-H in the paste, and therefore to an increasing amount of water adsorbed in its pores. Vice versa, the water adsorbed at $\mathrm{RH}=1$ accounts for all the pores in the system: leftover capillary pores plus nanopores inside the $\mathrm{C}-\mathrm{S}-\mathrm{H}$. An increasing degree of hydration causes the filling of some capillary pores, thus the maximum volume of water that can be adsorbed in the capillary plus the nano pores decreases overall.

For the BNG mechanism in Figure 5, the solid volume fraction of the $\mathrm{C}-\mathrm{S}-\mathrm{H}$ domains and the nanopore volume fraction in the $\mathrm{C}-\mathrm{S}-\mathrm{H}$ not change with time. This dense $\mathrm{C}-\mathrm{S}-\mathrm{H}$ has only small pores, smaller than the particle diameter of $10 \mathrm{~nm}$ because the particles are closely packed: as a result the isotherm has a clear finite slope only at $\mathrm{RH}$ between 0.2 and 0.7, which the Kelvin equation associates to pore sizes between 1 and $10 \mathrm{~nm}$. The isotherm is then almost flat for $\mathrm{RH}>0.8$, which is unrealistic because experimental water sorption isotherms always display a finite slope also for $\mathrm{RH}>0.8$ (Baroghel-Bouny 2007).

The homogeneous gelation mechanism and the densification mechanisms yield instead similar and more realistic results, in that both contain a wide range of pore sizes within the $\mathrm{C}-\mathrm{S}-\mathrm{H}$ between the nanometre and the micrometre, which evolve during hydration. This suggest that a gel densification mechanisms is necessary in order to predict realistic water sorption isotherms and related pore size distributions.

\section{CONCLUSION}

Nanoparticle-based simulations have indicated two different mechanisms of $\mathrm{C}-\mathrm{S}-\mathrm{H}$ precipitation: a homogeneus nucleation and growth (gelation) leading to a low density but densifying morphology, and a boundary nucleation and growth (BNG) leading to a high density hemispherical morphoology. These two mechanisms have been employed in simple macroscale simulations of cement hydration, which provide the evolution of water content and pore size distribution in the paste as hydration progresses.

The results show that the nanoscale morphology of the $\mathrm{C}-\mathrm{S}-\mathrm{H}$ has a significant impact on self-desiccation and water sorption isotherms. In particular, the BNG mechanism predicts no self desiccation until an unrealistically high degree of hydration is reached. The homogeneous gelation mechanism instead predicts realistic self-desiccation at sufficiently large degrees of hydration, $c a$. 0.4 and above, but has two shortfalls: firstly, it predicts an early onset of self-desiccation at too low degree of hydration ( $c a .0 .25$ instead of the experimental 0.4), secondly, it entails proportionality between degree of hydration and water to cement ratio, also not recorded in the experiments. The results thus point to an intermediate mechanism, with the $\mathrm{C}-\mathrm{S}-\mathrm{H}$ forming initially at low density but following the heterogeneous nucleation of $\mathrm{BNG}$, and then densifying in time in a similar manner as observed in the homogeneous gelation mechanisms. This intermediate mechanism, informed by experimentally measured evolutions of $\mathrm{C}-\mathrm{S}-\mathrm{H}$ density during hydration and implemented in a simple hydration model, realistically predicts both self-desiccation and water sorption isotherms.

Overall, these results indicate that: (i) macroscopic simulators of cement hydration should account for the nanoscale morphology and precipitation mechanisms of the hydration products; (ii) the nanoscale mechanisms and morphology of the $\mathrm{C}-\mathrm{S}-\mathrm{H}$ may be tuned to nanoengineer the interaction between cement paste and water, with potential for improving our control on the durability of concrete.

\section{REFERENCES}

Baroghel-Bouny, V. (2007). Water vapour sorption experiments on hardened cementitious materials: part I: essential tool for analysis of hygral behaviour and its relation to pore structure. Cem. Concr. Res. 37(3), 414 437.

Bažant, Z., A. Donmez, E. Masoero, \& S.R. Aghdam (2015). Interaction of concrete creep, shrinkage and swelling with water, hydration, and damage: Nanomacro-chemo. In CONCREEP 10, pp. 1-12.

Bullard, J.W., H.M. Jennings, R.A. Livingston, A. Nonat, G.W. Scherer, J.S. Schweitzer, K.L. Scrivener, \& J.J. Thomas (2011). Mechanisms of cement hydration. Cem. Concr. Res. 41, 1208-1223.

Bullard, J.W., G.W. Scherer, \& J.J. Thomas (2015). Time dependent driving forces and the kinetics of tricalcium silicate hydration. Cement and Concrete Research 74, 26-34.

Cusatis, G., M. Pathirage, G. Di Luzio, E. Masoero, \& D. Bentz (2018). A multiscale framework for the prediction of concrete hygral, thermal, and mechanical properties. In Computational Modelling of Concrete and Concrete Structures.

Davie, C. \& E. Masoero (2015). Modelling damage from the nano-scale up. In CONCREEP 10, pp. 613-620.

Del Gado, E., K. Ioannidou, E. Masoero, A. Baronnet, R.-M. Pellenq, F.-J. Ulm, \& S. Yip (2014). A soft matter in construction-statistical physics approach to formation and mechanics of $\mathrm{c}-\mathrm{s}-\mathrm{h}$ gels in cement. The European Physical Journal Special Topics 223(11), 2285-2295.

Di Luzio, G. \& G. Cusatis (2009). Hygro-thermo-chemical modelling of high performance concrete. i: Theory. Cement and Concrete composites 31(5), 301-308.

González-Teresa, R., V. Morales-Florez, H. Manzano, \& J. Dolado (2010). Structural models of randomly 
packed Tobermorite-like spherical particles: A simple computational approach. Materiales de Construcción 60(298), 7-15.

Ioannidou, K., K.J. Krakowiak, M. Bauchy, C.G. Hoover, E. Masoero, S. Yip, F.-J. Ulm, P. Levitz, R.J.M. Pellenq, \& E. Del Gado (2016). Mesoscale texture of cement hydrates. Proceedings of the National Academy of Sciences 113(8), 2029-2034.

Ioannidou, K., R.J.-M. Pellenq, \& E. Del Gado (2014). Controlling local packing and growth in calciumsilicate-hydrate gels. Soft Matter, 1121-1133.

Jennings, H.M., E. Masoero, M.B. Pinson, E.G. Strekalova, P.A. Bonnaud, H. Manzano, Q. Ji, J.J. Thomas, R.J.-M. Pellenq, F.-J. Ulm, et al. (2013). Water isotherms, shrinkage and creep of cement paste: hypotheses, models and experiments. In Mechanics and Physics of Creep, Shrinkage, and Durability of Concrete: A Tribute to Zdeňek P. Bažant, pp. 134-141.

Lasaga, A.C. (2014). Kinetic theory in the earth sciences. Princeton University Press.

Masoero, E., G. Cusatis, \& G. Di Luzio (URev). CSH gel densification: the impact of the nanoscale on self desiccation and sorption isotherms.

Masoero, E., E. Del Gado, R.J.-M. Pellenq, F.-J. Ulm, \& S. Yip (2012). Nanostructure and nanomechanics of cement: Polydisperse colloidal packing. Physical Review Letters 109(15), 155503.

Masoero, E., E. Del Gado, R.J.-M. Pellenq, S. Yip, \& F.-J. Ulm (2014). Nano-scale mechanics of colloidal C-S-H gels. Soft Matter 10, 491-499.

Masoero, E., H. Manzano, E. Del Gado, R. Pellenq, F. Ulm, \& S. Yip (2013). Kinetic simulation of the logarithmic creep of cement. In Mechanics and Physics of Creep, Shrinkage, and Durability of Concrete: A Tribute to Zdenek P. Bažant, pp. 166-173. ASCE.

Masoero, E., J.J. Thomas, \& H.M. Jennings (2014). A reaction zone hypothesis for the effects of particle size and water-to cement ratio on the early hydration kinetics of C3S. Journal of the American Ceramic Society 97(3), 967-975.

Muller, A., K. Scrivener, A. Gajewicz, \& P. McDonald (2013). Use of bench-top NMR to measure the density, composition and desorption isotherm of $\mathrm{C}-\mathrm{S}-\mathrm{H}$ in cement paste. Microporous and Mesoporous Materials 178(0), 99-103.

Muller, A.C.A. (2014). Characterization of porosity \& CSH in cement pastes by $1 H$ NMR. Ph.D. thesis, Ecole Polytechnique Federale De Lausanne.

Shvab, I., L. Brochard, H. Manzano, \& E. Masoero (2017). Precipitation mechanisms of mesoporous nanoparticle aggregates: off-lattice, coarse-grained, kinetic simulations. Crystal Growth \& Design 17(3), 1316-1327.

Tajuelo Rodriguez, E., I. Richardson, L. Black, E. Boehm- Courjault, A. Nonat, \& J. Skibsted (2015). Composition, silicate anion structure and morphology of calcium silicate hydrates (csh) synthesised by silica-lime reaction and by controlled hydration of tricalcium silicate (c3 s). Advances in Applied Ceramics 114(7), 362-371.

Thomas, J.J. (2007). A new approach to modeling the nucleation and growth kinetics of tricalcium silicate hydration. J. Am. Ceram. Soc 90, 3282-3288. 